\title{
Erratum
}

\section{Dynamics of behavioral sensitization induced by the dopamine agonist quinpirole and a proposed central energy control mechanism}

\author{
Henry Szechtman ${ }^{1,2}$, Hazel Talangbayan ${ }^{1}$, Gitanjali Canaran ${ }^{1}$, Hugh Dai ${ }^{1}$, David Eilam ${ }^{3}$ \\ ${ }^{1}$ Departments of Biomedical Sciences, McMaster University, 1200 Main St West, Hamilton, Ontario, Canada L8N 375 \\ ${ }^{2}$ Departments of Psychiatry and Psychology, McMaster University, 1200 Main St West, Hamilton, Ontario, Canada L8N 3Z5 \\ ${ }^{3}$ Department of Zoology, Tel-Aviv University, Ramat Aviv, Israel
}

Received: 2 August 1993/ Accepted: 3 August 1993

In the above article the wrong figure was printed for Fig. 2 (bottom) on p. 97. The correct figure and legend are printed below.
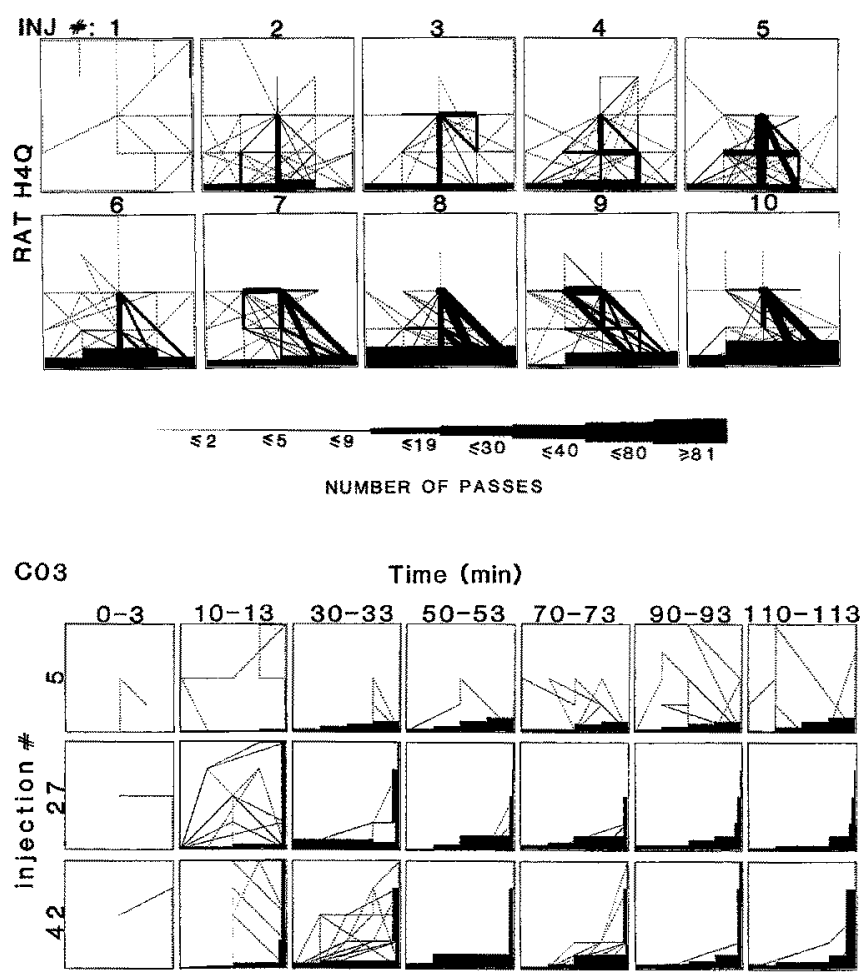

Fig. 2. Spatial distribution of paths of locomotion during the course of chronic treatment for two representative rats. Top panel depicts for one rat, for each of the first ten injections, the trajectories of locomotion summed across six time samples from 10 to $110 \mathrm{~min}$ after treatment. The thicker the line, the more often the rat locomoted between the indicated places on the open field, as shown on the scale. Bottom panel illustrates for one rat the trajectories of locomotion in all 3-min samples during the course of drug action for quinpirole injections 5,27 , and 42 (one injection per row). Note the stability of the preference to locomote along the same portion of the environment during the course of the $2 \mathrm{~h}$ of observation, and from injection to injection (spanning a period of about 9 weeks) 